\title{
Evaluating perfusion of thoracic spinal cord blood using CEUS during thoracic spinal stenosis decompression surgery
}

\author{
J Ling $^{1}$, W Jinrui ${ }^{1}$, C Ligang ${ }^{1}, \mathrm{C}$ Wen ${ }^{1}$, L Xiaoguang ${ }^{2}$ and J Liang ${ }^{2}$
}

Study design: A clinical study in human spinal cord.

Objectives: To evaluate changes in spinal cord blood perfusion in patients with thoracic spinal stenosis using contrast-enhanced ultrasonography and to semiquantitatively analyze blood perfusion changes in compressed spinal cord before and after ventral decompression.

Setting: Ultrasound department of a university hospital.

Methods: Twelve patients with confirmed thoracic spinal stenosis who needed decompression surgery participated. They underwent an intravenous injection of a contrast agent before and after ventral decompression. Quantitative analysis software (Philips Healthcare, Bothell, WA, USA) was used to perform time-intensity curve (TIC) analysis. The enhanced intensity $(\Delta I)$, rise time $(\Delta T)$ and slope of the TIC $(\beta)$ were separately calculated; $t$-tests of the independent samples were performed on the indicators.

Results: The TICs showed no significant differences between compressed spinal cord and normal spinal cord in $\Delta T$, enhanced $\Delta /$ and $\beta(P=0.46, P=0.23$ and $P=0.16$, respectively). After ventral decompression, $\Delta /$ of the originally compressed spinal cord increased substantially $(P=0.04)$ compared with $\Delta /$ of the normal spinal cord; however, the $\Delta T$ and $\beta$ showed no significant differences ( $P=0.18$ and $P=0.09$, respectively). Comparison of the blood perfusion parameters (that is, $\Delta T$ and $\Delta /$ ) of the compressed spinal cords before and after ventral decompression showed no significant differences $(P=0.14$ and $P=0.12$, respectively), but $\beta$ showed significant difference $(P=0.02)$.

Conclusion: Contrast-enhanced ultrasonography can dynamically display spinal cord blood perfusion. The characteristics of blood perfusion can be semiquantitatively analyzed using a software technique.

Spinal Cord (2015) 53, 195-199; doi:10.1038/sc.2014.213; published online 13 January 2015

\section{INTRODUCTION}

Two primary reasons thoracic spinal stenosis can cause spinal cord injury are chronic spinal cord compression and obstacles to spinal cord blood supply. Most experts believe that the perfusion state of spinal cord blood is closely correlated with the extent of spinal cord injury and the recovery of spinal functions after an operation. ${ }^{1}$

Three common methods of studying spinal cord blood perfusion are (1) the hydrogen clearance method, (2) isotope labeling and (3) magnetic resonance imaging (MRI). ${ }^{2}$ The first two methods can relatively accurately measure the local blood flow of the spinal cord. However, they can only be applied to animal models. In recent years, with the development of medical imaging, some studies have reported measuring blood volume with new MRI technology. ${ }^{3}$ However, because of low spatial resolution and bone artifacts, it too can only be applied to animal models.

Color Doppler blood flow imaging, which is widely used in clinical fields, can clearly show parenchymal organs and their internal blood flow. However, very little Doppler blood flow information is available when observing the blood perfusion of the spinal cord. The imaging technique contrast-enhanced ultrasonography (CEUS) shows the microvessels of organs by using the intravenous injection of microbubbles as the contrast agent. ${ }^{4}$ The diameter of the microbubbles is smaller than that of the red blood cells; therefore, they can smoothly pass by blood vessels without penetrating into the tissue space. Furthermore, the high frame rate real-time imaging ability of ultrasound makes it possible to display in real time the dynamic blood flow and tissue perfusion features of the tiny blood vessels in the body. In recent years, some studies that used ultrasound contrast agents described changes in blood flow perfusion of the spinal cord after acute spinal cord injury in animal models. ${ }^{5}$ However, no literature report exists on the evaluation of spinal cord blood flow (SCBF) perfusion using ultrasound contrast agents. Therefore, the current study used real-time gray-scale CEUS to evaluate SCBF perfusion during an operation.

\section{MATERIALS AND METHODS \\ Study participants \\ Between 1 January 2011 and 31 December 2011, 12 patients with thoracic spinal stenosis were admitted into our hospital. Five patients were male and seven were female. The average age was $50.83 \pm 9.2$ years. All patients underwent a clinical examination, which included anterior-posterior and lateral X-ray imaging, and computed tomography (CT) or MRI scans. They were confirmed as having severe thoracic spinal stenosis. Ten patients also had}

\footnotetext{
${ }^{1}$ Ultrasound Diagnosis Department, Peking University Third Hospital, Beijing, China and ${ }^{2}$ Department of Osteology, Peking University Third Hospital, Beijing, China Correspondence: Dr W Jinrui, Ultrasound Diagnosis Department, Peking University Third Hospital, North Garden Road 49th, Haidian District, Beijing 100191, China. E-mail: usjinrui@126.com or L Xiaoguang, Department of Osteology, Peking University Third Hospital, North Garden Road 49th, Haidian District, Beijing 100191, China. E-mail: xglius@vip.sina.com

Received 29 May 2014; revised 29 September 2014; accepted 16 October 2014; published online 13 January 2015
} 
ossification of the posterior longitudinal ligament. One patient also had diffuse idiopathic skeletal hyperostosis. A $360^{\circ}$ circular decompression was conducted on 20 thoracic cord segments in the 12 patients. Before the operation, the patients were scored by the 11-score method of the Japanese Orthopaedics Association scoring system. The patients' Japanese Orthopaedics Association score was $4.54 \pm 1.96$ points (range, $0-7$ points). The follow-up period was 3-6 months. CT scans or MRI scans were used for evaluation.

\section{Instruments and equipment}

Color Doppler ultrasonic diagnostic equipment (Philips iU22 ultrasound system; Philips Healthcare) was used to assess the 12 patients. The L9-3 probe used pulse-inverse harmonic imaging and built-in analysis software (QLAB9.0, Seattle, WA, USA). Imaging is achieved under the default settings (that is, $\mathrm{MI}=0.09$ ). The other settings (for example, the depth of the scan, overall gain, time gain compensation and dynamic range) remained constant during the imaging process.

\section{Contrast agent}

The contrast agent was SonoVue (Bracco, Milan, Italy). The microbubbles ranged $1-10 \mu \mathrm{m}$ in diameter with an average diameter of $\sim 2.5 \mu \mathrm{m}$ and $90 \%$ of the microbubbles had a diameter $<6 \mu \mathrm{m}$ and $99 \%$ of the microbubbles had a diameter $<11 \mu \mathrm{m}$. The contrast agent was prepared in $5-\mathrm{ml}$ microbubble suspensions.

\section{Study method}

All patients underwent $360^{\circ}$ circumferential decompression of the spinal cord, called the 'cave-in technique,' which was created by our hospital. ${ }^{6}$ In this technique ventral and dorsal decompression of the thoracic cord is performed simultaneously during surgery. When the patient was well under general anesthesia, the patient's body was turned over to a prone position. The space under the stomach was padded. A median incision was created on the chest and back to reveal the decompressed thoracic segments and the spinous processes and vertebral plates of the first two segments at both ends. The 'cap uncovering method' was used to remove the back wall of the canalis spinalis, and normal saline was infused to form an acoustic window through which ultrasonic examinations were performed to observe the spinal form.

The normal dural surface appears as a smooth hyperechoic line within which are isoechoic bands of spinal structures with a banding anechoic area on both sides of the spinal cord. This indicates flowing cerebrospinal fluid.

The compression segment of the dural sac and the spinal cord is a significant finding with compression of a hyperechoic structure ventrally (Figure 1). If the

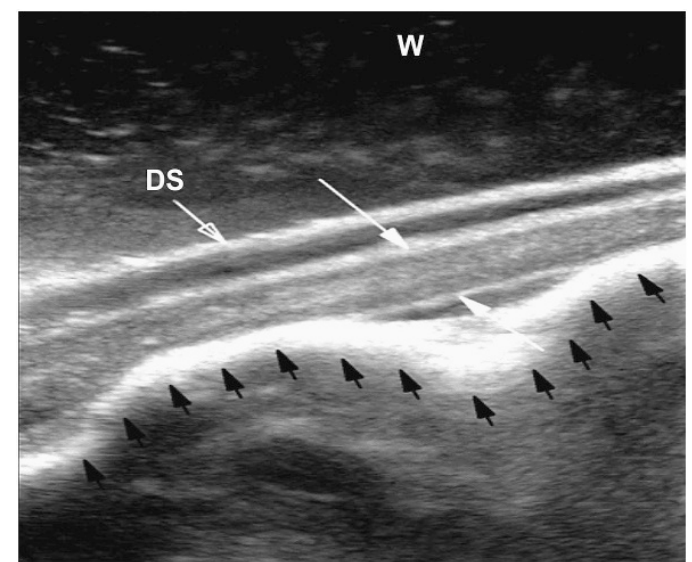

Figure 1 The rear wall of the spinal canal is removed and longitudinal inspection is conducted using normal saline as the acoustic window. The dural sac (hollow white arrow) and ossified posterior longitudinal ligament (black solid arrows) compress the spinal cord (solid white arrows). DS =dural sac; $W=$ normal saline ventral compression of the spinal cord remained, the following examination was required.

Contrast agent $(2.5 \mathrm{ml})$ was injected through the elbow vein group and the blood vessels were immediately washed with $5 \mathrm{ml}$ of normal saline. An L9-3 broadband linear-array probe was used to collect and save real-time continuous and dynamic ultrasonographic images from the initiation of the injection to $1 \mathrm{~min}$ after the completion of the injection. When performing ventral decompression of the spinal cord (Figure 2), a contrast examination is again conducted as per the previous method. Figure 3 shows CT scans of the same patient before and after annular decompression surgery.

\section{Image analysis}

The original dynamic images stored on the hard disk of the IU22 equipment were copied and an offline analysis on the machine was conducted. The QLAB analysis software was started and 2 ROIs were placed on the compressed spinal cord and on the normal spinal cord. The normal spinal cord was the portion of

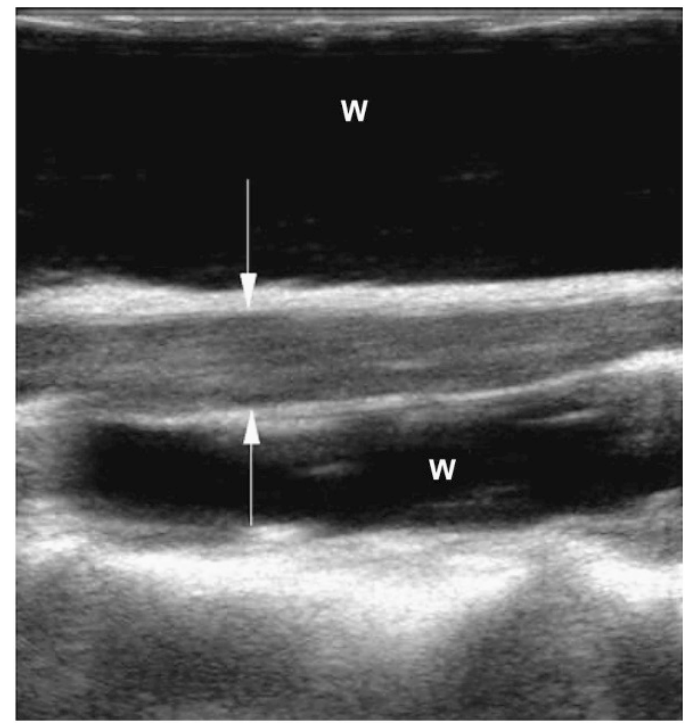

Figure 2 The ultrasonic longitudinal scan during the operation shows that the dural sac and the spinal cord (solid white arrow) have returned to their normal forms after circular decompression. $\mathrm{W}=$ normal saline.
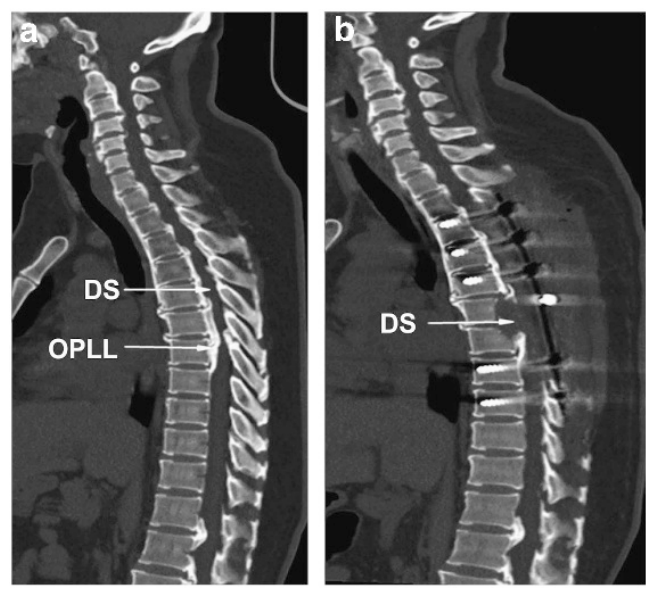

Figure 3 (a) The computed tomography scan before the operation clearly shows that the ossified posterior longitudinal ligament (OPLL) squeezes the dural sac (DS). (b) In the same patient, the local DS is not compressed after circular decompression. 
the spinal cord with a normal form that was $1 \mathrm{~cm}$ away from the compressed site cranially or caudally. The position and the scope were basically the same. Commonly used blood perfusion parameters were obtained, which included the following: the time to start developing (that is, arrival time); the rise time $(\Delta T)$, which is the difference between the values of the rise time and the time to start developing; enhanced intensity $(\Delta I)$, which is the difference between values of the peak intensity and background intensity; and the slope of the curve $(\beta)$, which is the ratio of $\Delta I$ to $\Delta T$.

\section{Statistical analysis}

The ultrasound images of the 12 patients were converted into time-intensity curves (TICs). The spinal cord blood perfusion parameters (that is, $\Delta T, \Delta I$ and $\beta$ ) of the compressed spinal cord before and after decompression were obtained and compared with these parameters in the normal spinal cord. The numerical variables are denoted by $x \pm s$. The statistical data were analyzed with the independent samples $t$-test by using SPSS17.0 software (SPSS, Chicago, IL, USA). The difference was significant at $P<0.05$. Parameters of the compressed spinal cords before and after ventral decompression were analyzed with a paired t-test. The difference was significant at $P<0.05$.

Statement of ethics. We certify that all applicable institutional and governmental regulations concerning the ethical use of human volunteers were followed during the course of this research. The study passed the ethical review of the Medical Ethics Committee of the Peking University Third Hospital (Beijing, China). Every patient signed the informed consent form. The project number is IRB00006761-2011094.

\section{RESULTS}

In the 12 patients, the microbubbles reached the spinal cord $23.68 \pm 6.14 \mathrm{~s}$ after the intravenous injection of the contrast agent. The microbubbles were blocked and moved slowly through the compressed spinal cord (i.e., the road is 'open but not smooth'). The TIC was relatively straight (the red curve in Figure 4). After complete decompression, the microbubbles in the spinal cord could pass freely. The slope of the TIC increased and became relatively steep (the red curve in Figure 5).

Before ventral decompression, the TICs of the normal spinal cord and the compressed spinal cord were obtained with the following parameters: $\Delta I, \Delta T$ and $\beta$ (Table 1). The results showed no significant difference between the normal and compressed spinal cords with regard to the aforementioned blood parameters $(P=0.46$ for $\Delta T$; $P=0.23$ for $\Delta I$; and $P=0.16$ for $\beta$ ).

After ventral decompression, the TICs of the normal spinal cord and the compressed spinal cord and the parameters $\Delta \mathrm{I}, \Delta T$ and $\beta$ were obtained (Table 2). There was a significant difference between the normal and compressed spinal cords only in $\Delta I(P=0.04)$. There was no significant difference in $\Delta T$ and $\beta(P=0.18$ and $P=0.09$, respectively).

The parameters $\Delta I, \Delta T$ and $\beta$ of the compressed spinal cords before and after ventral decompression were obtained (Table 3). $\Delta T$ and $\Delta I$ showed no significant differences $(P=0.14$ and $P=0.12$, respectively), whereas $\beta$ showed a significant difference $(P=0.02)$.

\section{DISCUSSION}

Evaluating the lesion and the blood flow perfusion of organic tissues by combining real-time gray-scale CEUS with newer ultrasonic contrast agents has been successfully applied to abdominal organs. The current study evaluated the blood flow perfusion of the spinal cord during decompression by administering a peripheral intravenous injection of ultrasonic contrast agents to patients with thoracic spinal stenosis.

After removing the dorsal vertebral plate, longitudinal scanning was conducted on the long axis of the spinal cord to perform ultrasonography. The study showed that the microbubbles arrived at the spinal cord of all patients $23.28 \pm 6.14 \mathrm{~s}$ after the injection. For the primary purpose of this study and the division of the regions of interest (ROIs),

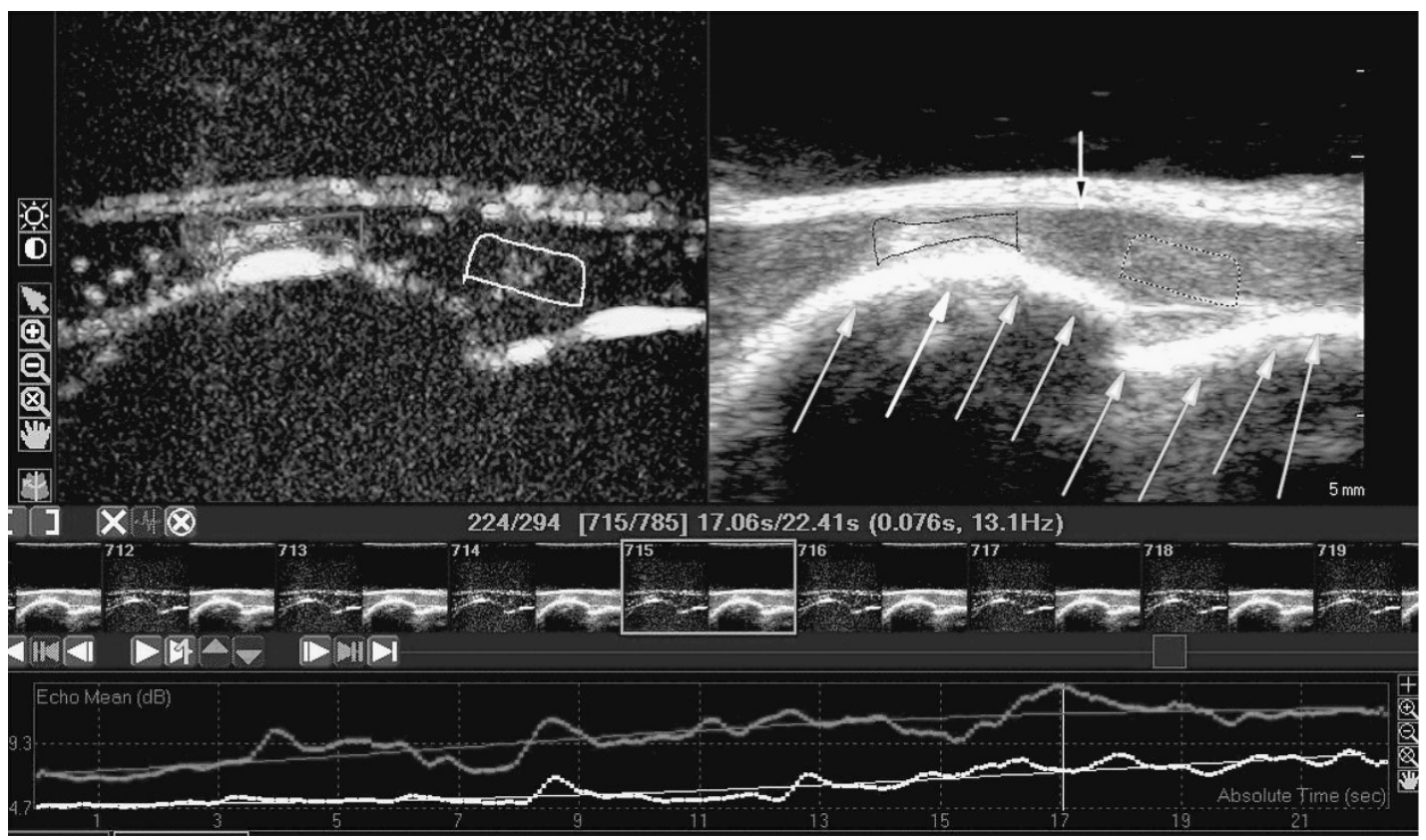

Figure 4 The longitudinal scan of the spinal cord after removal of the spinal lamina. The left side of the figure is the contrast image and the right side is the corresponding 2-dimensional acoustic image. The 2-dimensional image shows the posterior longitudinal ligament (white arrow) squeezing with strong echoes at the ventral side of the compressed spinal cord (black arrow). The regions of interest (ROIs) for the compressed spinal cord are outlined in red and the corresponding time-intensity curve (TIC) is relatively straight (red line on the bottom). The ROls for the normal spinal cord are outlined in yellow, and the corresponding TIC is obtained (yellow line on the bottom). A full color version of this figure is available at the Spinal Cord journal online. 


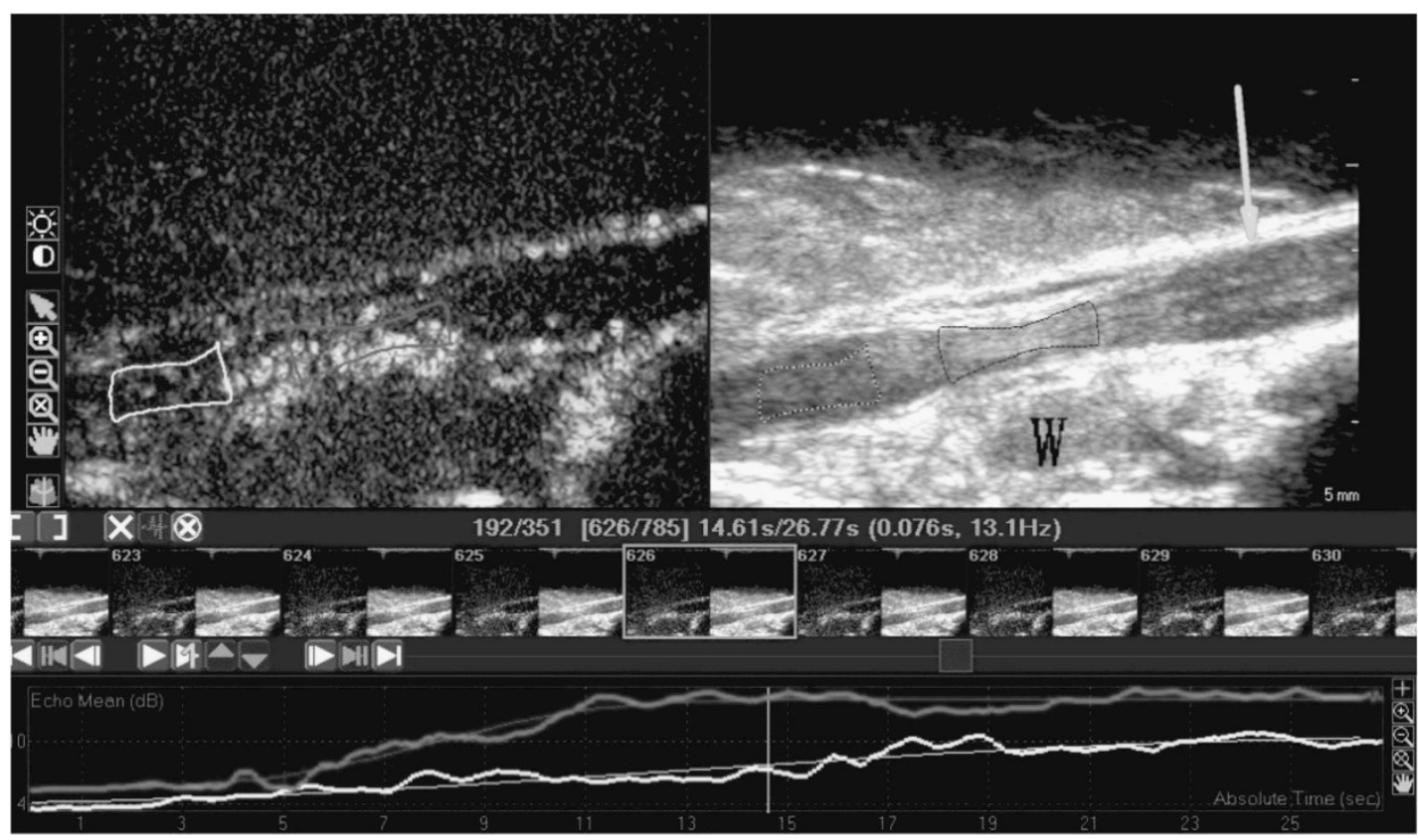

Figure 5 The longitudinal scan of the spinal cord after circular decompression in the same patient. The left side of the figure is the contrast image and the right side of the figure is the corresponding 2-dimensional acoustic image. The ossified posterior longitudinal ligament at the ventral side of the original spinal cord (white arrow) is completely removed and is narrow. The deep square on the right image is normal saline (W). The regions of interest (ROIs) for the decompressed spinal cord are outlined in red, and the corresponding time-intensity curve (TIC) is relatively straight (red line on bottom). The ROI for the normal spinal cord is outlined in yellow and the corresponding TIC is obtained (yellow line on the bottom). W=normal saline. A full color version of this figure is available at the Spinal Cord journal online.

Table 1 Comparison between the compressed spinal cords and the normal spinal cords in the blood perfusion parameters before ventral decompression

\begin{tabular}{|c|c|c|c|c|c|}
\hline & $\begin{array}{c}\text { Case } \\
\text { number }\end{array}$ & $\begin{array}{c}\text { Compressed } \\
\text { spinal cord } \\
\quad(\mathrm{x} \pm \mathrm{s})\end{array}$ & $\begin{array}{c}\text { Normal } \\
\text { spinal cord } \\
(\mathrm{x} \pm \mathrm{s})\end{array}$ & $t$ & P-value \\
\hline Rise time, $\Delta T$ & 12 & $11.43 \pm 6.37$ & $13.24 \pm 5.64$ & -0.73 & 0.46 \\
\hline $\begin{array}{l}\text { Enhanced } \\
\text { intensity, } \Delta /\end{array}$ & 12 & $5.28 \pm 2.81$ & $4.23 \pm 1.04$ & 1.21 & 0.23 \\
\hline $\begin{array}{l}\text { Slope of } \\
\text { curve, } \beta\end{array}$ & 12 & $0.53 \pm 0.38$ & $0.34 \pm 0.19$ & 1.45 & 0.16 \\
\hline
\end{tabular}

it was necessary to avoid large and thick blood vessels on the spinal surface and focus primarily on the blood perfusion within the spinal parenchyma.

During the operation, the first ultrasonography scan was conducted after removing the vertebral plate. Because of physiologic kyphosis of the thoracic vertebrae, after solely removing the back wall of the thoracic spinal canal, the dural sac could not float backward as it would after the enlargement of the cervical spinal canal. ${ }^{7}$ The spinal cord remained in a state of ventral compression, but the degree of compression was obviously alleviated, compared with before the operation. The TIC of the compressed spinal cord in comparison with that of the nearby normal spinal cord showed no significant difference in intensity changes, enhancement duration or slopes of the TICs. This showed that no obvious difference exists between the compressed and normal spinal cords in perfusion speed and intensity. This is different from the common opinion that spinal cord ischemia is the main pathological and physiological mechanism occurring after
Table 2 Comparison between the compressed spinal cords and the normal spinal cords in the blood perfusion parameters after ventral decompression

\begin{tabular}{lcccrr}
\hline & $\begin{array}{c}\text { Case } \\
\text { number }\end{array}$ & $\begin{array}{c}\text { Compressed } \\
\text { spinal cord } \\
(\mathrm{x} \pm \mathrm{s})\end{array}$ & $\begin{array}{c}\text { Normal spinal } \\
\text { cord } \\
(\mathrm{x} \pm \mathrm{s})\end{array}$ & $t$ & P-value \\
\hline $\begin{array}{l}\text { Rise time, } \Delta T \\
\text { Enhanced }\end{array}$ & 12 & $7.96 \pm 4.38$ & $11.49 \pm 7.80$ & -1.37 & 0.18 \\
$\begin{array}{l}\text { intensity, } \Delta / \\
\text { Slope of }\end{array}$ & 12 & $8.52 \pm 4.93$ & $5.44 \pm 1.31$ & 2.09 & 0.04 \\
curve, $\beta$ & 12 & $1.39 \pm 1.20$ & $0.71 \pm 0.64$ & 1.72 & 0.09 \\
\hline
\end{tabular}

Table 3 Comparison of the blood perfusion parameters of the compressed spinal cords before and after ventral decompression

\begin{tabular}{lcccrc}
\hline \multicolumn{7}{c}{$\begin{array}{c}\text { Case } \\
\text { number }\end{array}$} & $\begin{array}{c}\text { Before ventral } \\
\text { decompression } \\
(\mathrm{x} \pm \mathrm{s})\end{array}$ & $\begin{array}{c}\text { After ventral } \\
\text { decompression } \\
(\mathrm{x} \pm \mathrm{s})\end{array}$ & $\mathrm{t}$ & P-value \\
\hline Rise time, $\Delta T$ & 12 & $11.43 \pm 6.37$ & $7.95 \pm 4.37$ & 1.594 & 0.14 \\
Enhanced intensity, $\Delta I$ & 12 & $5.28 \pm 2.80$ & $8.51 \pm 4.92$ & -1.700 & 0.12 \\
Slope of curve, $\beta$ & 12 & $0.53 \pm 0.38$ & $1.39 \pm 1.20$ & -2.641 & 0.02 \\
\hline
\end{tabular}

spinal cord injury. Most animal experiments show that, after a spinal cord injury, local vasospasm and microvascular rupture occur. ${ }^{8}$ Spinal injury can also lead directly to endothelial injury or swelling, and thus result in thrombosis formation in local tiny blood vessels and reduce blood flow perfusion. The current study findings showed that the 
spinal cord has a self-adjustment function when compression is not serious. Carlson et al. ${ }^{9}$ divided dog subjects into two groups. The first group had compressed spinal cords. The second group had undergone decompression of the spinal cords. Carlson then measured the volume of local SCBF using the fluorescent microsphere technique, and found that the blood volume of both groups after $3 \mathrm{~h}$ were virtually the same. Therefore, as in the cerebral cortex, the local blood flow of the spinal cord can regulate itself through changes in blood pressure and arterial partial pressure of carbon dioxide. ${ }^{10}$ The latest research shows that a baroreceptor may exist around the capillary bed of the anterior and dorsal spinal horns. ${ }^{11}$ The baroreceptor can detect tension due to muscle crush of the anterior spinal artery walls and thus directly regulate local blood flow of the spinal cord.

In this study, the second contrast-enhanced ultrasound scan was performed after spinal circular decompression to evaluate the blood flow perfusion of the compressed spinal cord after complete circular decompression. The injected contrast agent can be observed visually. The number of microbubbles is significantly larger for the decompressed spinal cord than for the normal spinal cord. A comparison of the TICs of the two cord groups showed that the intensity change for the compressed spinal cord was $8.52 \pm 4.93$ and that for the normal spinal cord was $5.44 \pm 1.31$. The difference between the former and the latter was significant $(P<0.05)$. This showed that the blood flow volume increases immediately after reducing the pressure on the spinal cord, which is manifested by the sign of congestion. The cause of the congestion may be the expansion of blood vessels after decompression and the decrease in resistance. According to relevant literature, ${ }^{12}$ after spinal cord injury, the local SCBF, vascular resistance and mean arterial blood pressure are closely correlated with each other $(\mathrm{SCBF}=$ mean arterial blood pressure /vascular resistance). During surgery, the mean arterial blood pressure remains relatively constant and the SCBF is inversely proportional to the vascular resistance. Local increased blood flow volume must be accompanied by vasodilatation. The vasodilatation may result from changes in ion concentration and the local release of bioactive substances such as histamine.

Previous studies on spinal cord ischemia-reperfusion are mostly confined to animal experiments that induced acute spinal cord ischemia by ligating the abdominal aorta of animals ${ }^{13}$ or by gradually inserting screws into the vertebral plate to compress the dorsal spinal cord. ${ }^{14}$ The aforementioned animal models are not fully consistent with actual situations, and the time windows for observing spinal cord perfusion are different. Therefore, there are few reports of congestion after spinal decompression. Most reports in the literature suggest that the longer the duration of spinal cord compression, the less the blood flow reperfusion. ${ }^{15}$ However, in experiments on animals with spinal cords compressed by airbags, Kobrine et al. ${ }^{16}$ found that the congestion phenomenon could occur within $15 \mathrm{~min}$ after the decompression, and they speculated that the congestion phenomenon occurs only in moderate spinal cord injury. The research results are consistent with the findings of the current study.

After thorough decompression, microbubbles can freely pass through the spinal cord. This phenomenon can also help a surgeon determine the extent of decompression of the compressed spinal cord. The TIC is flat before ventral decompression. The slope increases after thorough decompression. This indicates the necessity of ventral decompression surgery as it can significantly improve perfusion of the spinal cord.

In conclusion, contrast-enhanced ultrasound applied during thoracic spinal stenosis decompression surgery dynamically shows real-time spinal cord perfusion under compression, after decompression and in the normal state. The features of spinal cord perfusion under different states can be semiquantitatively analyzed using software technology.

This study has some limitations. First, the number of patients was small; thus, the perfusion features of the SCBF could not be correlated with the recovery status of neurological function. Second, the features of SCBF perfusion cannot be associated with pathology because spinal cord specimens of the patients could not be obtained.

\section{DATA ARCHIVING}

There were no data to deposit.

\section{CONFLICT OF INTEREST}

The authors declare no conflict of interest.

1 Truumees E. Spinal stenosis: pathophysiology, clinical and radiologic classification. Instr Course Lect 2005; 54: 287-302.

2 Kang CE, Clarkson R, Tator CH, Yeung IW, Shoichet MS. Spinal cord blood flow and blood vessel permeability measured by dynamic computed tomography imaging in rats after localized delivery of fibroblast growth factor. J Neurotrauma 2010; 27: 2041-2053.

3 Duhamel G, Callot V, Cozzone PJ, Kober F. Spinal cord blood flow measurement by arterial spin labeling. Magn Reson Med 2008; 59: 846-854.

4 Cosgrove D. Ultrasound contrast agents: an overview original research article. Eur J Radiol 2006; 60: 324-330.

5 Soubeyrand M, Laemmel E, Dubory A. Real-time and spatial quantification using contrast-enhanced ultrasonography of spinal cord perfusion during experimental spinal cord injury. Spine (Phila Pa 1976) 2012; 37: E1376-E1382.

6 Wang YQ, Liu XG, Jiang L, Jiang L, Wei F, Yu M et al. Intraoperative ultrasonography in "cave-in" $360^{\circ}$ circumferential decompression for thoracic spinal stenosis. Chin Med J (Engl) 2011; 124: 3879-3885.

7 Yamazaki M, Okawa A, Fujiyoshi T, Furuya T, Koda M. Posterior decompression with instrumented fusion for thoracic myelopathy caused by ossification of the posterior longitudinal ligament. Eur Spine J 2010; 19: 691-698.

8 Koyanagi I, Tator $\mathrm{CH}$, Lea PJ. Three-dimensional analysis of the vascular system in the rat spinal cord with scanning electron microscopy of vascular corrosion casts. Part 2: acute spinal cord injury. Neurosurgery 1993; 33: 285-291.

9 Carlson GD, Warden KE, Barbeau JM, Bahniuk E, Kutina-Nelson KL, Biro CL et al. Viscoelastic relaxation and regional blood flow response to spinal cord compression and decompression. Spine (Phila Pa 1976) 1997; 22: 1285-1291.

10 Mautes AE, Weinzierl MR, Donovan F, Noble LJ. Vascular events after spinal cord injury: contribution to secondary pathogenesis. Phys Ther 2000; 80: 673-687.

11 Parke WW. Arteriovenous glomeruli of the human spinal cord and their possible functional implications. Clin Anat 2004; 17: 558-563.

12 Martirosyan NL, Feuerstein JS, Theodore N, Cavalcanti DD, Spetzler RF, Preul MC. Blood supply and vascular reactivity of the spinal cord under normal and pathological conditions. J Neurosurg Spine 2011; 15: 238-251.

13 Etz CD, Homann TM, Luehr M, Kari FA, Weisz DJ, Kleinman G et al. Spinal cord blood flow and ischemic injury after experimental sacrifice of thoracic and abdominal segmental arteries. Eur J Cardiothorac Surg 2008; 33: 1030-1038.

14 Xu P, Gong WM, Li Y, Zhang T, Zhang K, Yin DZ et al. Destructive pathological changes in the rat spinal cord because of chronic mechanical compression. Laboratory investigation. J Neurosurg Spine 2008; 8: 279-285.

15 Gregory D, Carlson GD, Minato Y, Okada A, Warden KE, Barbeau JM et al. Early timedependent decompression for spinal cord injury: vascular mechanisms of recovery. $J$ Neurotrauma 1997; 14: 951-962.

16 Kobrine AI, Evans DE, Rizzoli HV. Correlation of SCBF, sensory evoked response, and spinal cord function in subacute experimental spinal cord compression. Adv Neurol 1978; 20: 389-394. 